\title{
The underlying terahertz vibrational spectrum of explosives solids
}

\author{
Joseph S. Melinger, ${ }^{1, a)}$ N. Laman, ${ }^{2}$ and D. Grischkowsky ${ }^{2}$ \\ ${ }^{1}$ Naval Research Laboratory, Electronics Science and Technology Division, Code 6812, Washington, DC \\ 20375, USA \\ ${ }^{2}$ School of Electrical and Computer Engineering, Oklahoma State University, Stillwater, Oklahoma 74078, \\ USA
}

(Received 9 May 2008; accepted 30 May 2008; published online 8 July 2008)

\begin{abstract}
Using waveguide terahertz time-domain spectroscopy, we demonstrate the measurement of the underlying terahertz vibrational spectrum of the explosive solids hexahydro-1,3,5-trinitro-1,3,5triazine, and 2,4,6-trinitrotoluene. Each explosive is cast as a polycrystalline thin film with planar ordering on the inner surface of metal parallel plate waveguide. For measurements near $10 \mathrm{~K}$, each explosive reveals a complex spectrum of approximately 20 vibrational modes between 0.5 and 3.5 THz. The explosive films are of sufficient quality to produce vibrational linewidths as much as an order of magnitude sharper compared to conventional terahertz measurements of corresponding pellet samples of explosives. (C) 2008 American Institute of Physics. [DOI: 10.1063/1.2949068]
\end{abstract}

One of the most promising and discussed applications of terahertz $(\mathrm{THz})$ spectroscopy is the detection and identification of explosive materials. ${ }^{1-3}$ Previous work has measured the $\mathrm{THz}$ absorption of explosive solids, revealing a number of broad features associated with the signature vibrational modes of the material. ${ }^{1,2,4-9}$ However, the majority of this work has been performed on disordered polycrystalline pellet samples, ${ }^{1,2,4-8}$ resulting in inhomogeneous broadening of the absorption features, making their identification more difficult. We have addressed this problem by developing the technique of waveguide terahertz time-domain spectroscopy (THz-TDS), ${ }^{10,11}$ whereby an ordered polycrystalline film is formed on a metal surface and its $\mathrm{THz}$ vibrational response is characterized via a parallel plate waveguide (PPWG). ${ }^{12,13}$ The planar order of the sample together with the formation of microcrystals of high crystalline quality reduces the inhomogeneous broadening, resulting in considerably sharper and much more identifiable features. ${ }^{11,14-18}$ Here, we present the application of waveguide THz-TDS to the explosive solids hexahydro-1,3,5-trinitro-1,3,5-triazine (RDX) and 2,4,6trinitrotoluene (TNT), resulting in a dramatic sharpening of the vibrational absorption lines as the films are cooled to near $10 \mathrm{~K}$. The linewidths measured at their full width at half maximum (FWHM) by waveguide THz-TDS are in some cases more than an order of magnitude sharper than corresponding $\mathrm{THz}$ measurements with standard pellets of explosives, and reveal a rich spectrum of approximately 20 narrow vibrational absorption lines between 0.5 and $3.5 \mathrm{THz}$. This information can provide an extraordinary precise "spectral fingerprint" capable of identification by a series of unique vibrational absorption lines. Such narrow vibrational lines can also provide rigorous input to help guide theoretical modeling ${ }^{19}$ and advance the understanding of the vibrational properties of explosive solids.

The THz measurement apparatus, method for the recovery of absorbance spectra for the waveguide films, and details of the PPWG have been described previously in Refs. 11 and 14. Prior to film formation, the metal waveguide plates were thoroughly washed with solvent and then plasma cleaned. Thin films of RDX and TNT were formed on the inner surface of an aluminum $(\mathrm{Al})$ or gold $(\mathrm{Au})$-coated $\mathrm{Al}$

\footnotetext{
${ }^{a)}$ Electronic mail: joseph.melinger@nrl.navy.mil.
}

waveguide plate by simple drop casting from an explosive standard solution (obtained from Accustandards Inc. and Sigma Aldrich, respectively) containing $1 \mathrm{mg} / \mathrm{ml}$ of explosive (99\% purity) in 1:1 acetonitrile:methanol, or in acetonitrile, respectively. For RDX, $150 \mu \mathrm{l}$ were dropcast onto the waveguide plate. For TNT it was necessary to concentrate the solution to $2.5 \mathrm{mg} / \mathrm{ml}$, followed by drop casting $200 \mu \mathrm{l}$ onto the plate. The rough edges of the films were swabbed away using a solvent soaked swab to yield a visibly uniform film. The coated waveguide plate was then incorporated into the PPWG. The masses of the RDX and TNT films are estimated to be approximately 150 and $300 \mu \mathrm{g}$ respectively.

Figures 1(a) and 1(b) show the spectral amplitudes transmitted through the PPWG containing RDX on Al and TNT on $\mathrm{Au}$, respectively, and measured as a function of temperature. The vibrational center frequencies and FWHM linewidths (for sufficiently resolved lines) resulting from the waveguide THz-TDS measurements are collected in Table I. The dips that appear in the spectral amplitudes are associated with vibrational absorptions of each film.

At room temperature, the spectral amplitude of the RDX film [Fig. 1(a)] shows relatively weak and broad absorption features. The most prominent absorption dip occurs near $0.83 \mathrm{THz}$, in agreement with previous free space $\mathrm{THz}$ measurements. ${ }^{5-7}$ To better clarify the known room temperature absorption features, we have also characterized a thicker RDX film on $\mathrm{Al}$, which is given in our supplementary material. ${ }^{20}$ As the RDX film is cooled, the broad absorption features present at $293 \mathrm{~K}$ undergo a pronounced sharpening, and many additional absorption features begin to appear. We also note that the vibrational lines exhibit a temperaturedependent frequency shift resulting from a thermal compression of the crystalline lattice ${ }^{21}$ and from anharmonicity of the vibrational potentials. ${ }^{21}$ At $80 \mathrm{~K}$, at least ten absorption features appear from 0.5 to $3.5 \mathrm{THz}$. As the film is further cooled to $13 \mathrm{~K}$, the absorption features continue to sharpen and in some cases split, leading to the observation of up to 19 vibrational lines (Table I). The FWHM linewidths of the sharpest vibrational lines approach our instrument resolution of $8 \mathrm{GHz}$ corresponding to the 133 ps temporal scans, and suggesting that their intrinsic linewidths could be substantially narrower. The relatively broad absorption at $1.04 \mathrm{THz}$ $(\mathrm{FWHM}=0.090 \mathrm{THz}$ at $13 \mathrm{~K}$ ) appears to be an exception, 


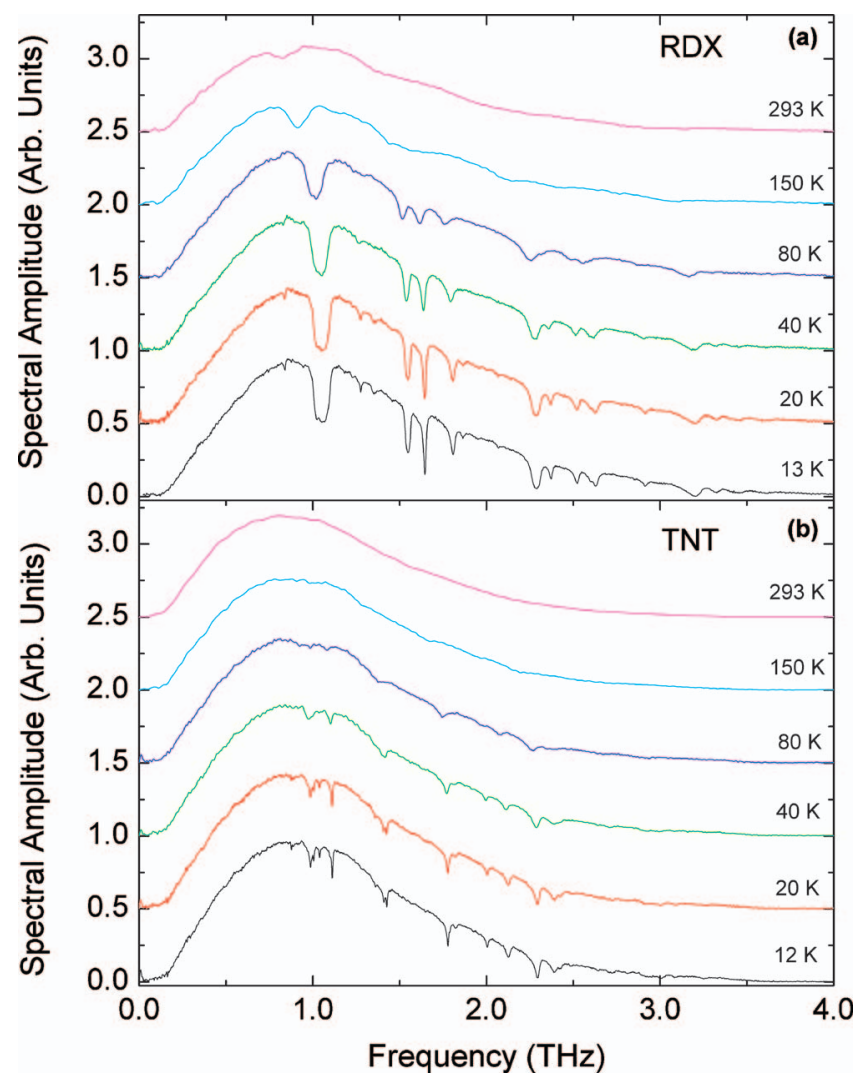

FIG. 1. (Color) Spectral amplitudes measured for RDX and TNT films in a metal PPWG as a function of temperature. (a) The RDX film on Al. (b) The TNT film on Au. The spectral amplitudes from 20 through $293 \mathrm{~K}$ are offset for clarity.

spectrally narrowing by approximately a factor of 2 relative to its room temperature linewidth $(0.14 \mathrm{THz}$ FWHM at a frequency of $0.83 \mathrm{THz}$ ). Close inspection of this lineshape suggests that it contains a superposition of several closely spaced individual lines.

The TNT film on Au [Fig. 1(b)] also displays strong line narrowing with cooling. At $293 \mathrm{~K}$, the spectral amplitude shows only weak and unresolved absorption features in the region between 0.8 and $1.2 \mathrm{THz}$. The observation of $24 \mathrm{ab}-$ sorption lines at $12 \mathrm{~K}$ highlights a dramatic line narrowing effect for the TNT waveguide film. Some of the TNT vibrational absorption lines exhibit instrument-limited FWHM linewidths of $8 \mathrm{GHz}$ (Table I).

We note that RDX films on $\mathrm{Au}$ and TNT films on Al were also measured (not shown here) and showed only small differences in the spectra for the different metal substrates.

For both explosives, a large number of low frequency vibrational lines are anticipated in the $\mathrm{THz}$ region due to both intermolecular and intramolecular modes. For a molecular crystal with a unit cell containing $Z$ molecules there should be $6 Z-3$ optical phonons and $Z(3 N-6)$ internal modes, where $N$ is the number of atoms in the molecule. RDX crystallizes in space group $P b c a$ with $Z=8 .^{22}$ TNT crystallizes in at least two polymorphs, ${ }^{23}$ space group $P c a 2_{1}, Z=8$, and $P 2_{1} / a, Z=8$ where $P 2_{1} / a$ is the more stable form. Thus, 45 optical phonons are predicted corresponding to each of the space groups (21 infrared-active phonons for centrosymmetric space groups $P b c a$ and $P 2_{1} / a$ ), many of which will have resonances below $4 \mathrm{THz}$. In addition, intramolecular modes involving low frequency large amplitude vibrational motion may also occur below $4 \mathrm{THz}$. Author complimentary copy. Redistribution subject to AIP license or copyright, see http://apl.aip.org/apl/copyright.jsp
TABLE I. Line center frequencies (bold) and FWHM linewidths (in parenthesis) for RDX and TNT films. All units are in THz.

Explosive film Absorbance line frequencies at $12 \mathrm{~K}$ (bold text) and FWHM linewidths in parentheses ${ }^{\mathrm{a}}$

RDX on Al $\mathbf{0 . 8 3 9}(0.010), \mathbf{0 . 8 7 6}, \mathbf{1 . 0 4}(0.090), 1.276(0.009)$

$1.351(0.025), 1.550(0.028), 1.642(0.017)$,

1.806 (0.023), $\mathbf{1 . 8 6 4}(0.015), \mathbf{2 . 0 6 9}, \mathbf{2 . 2 9 0}(0.046)$,

$2.370(0.018), 2.524(0.025), \mathbf{2 . 6 0 3}, 2.625(0.028)$,

$2.91(0.027), 3.20(0.040), 3.32(0.042), 3.45$

TNT on Au $\quad 0.877(0.008), 0.987,1.004,1.038(0.009)$ 1.10,

$1.111(0.008), 1.360,1.409,1.426(0.010), 1.778(0.011)$,

1.82, $2.005(0.016), \mathbf{2 . 1 2 5}(0.025), \mathbf{2 . 2 9 5}(0.020)$,

2.39 (0.040), 2.42, $2.49(0.023), 2.64(0.018)$,

$2.72(0.050), 2.81,2.94,3.01,3.09,3.25(0.035)$

${ }^{\mathrm{a}}$ The uncertainty in the line frequency is $\pm 0.004 \mathrm{THz}$ where there are four significant figures given and $\pm 0.010 \mathrm{THz}$ where there are three significant figures given. The uncertainty in the linewidths is $\pm 10-15 \%$.

The effectiveness of waveguide THz-TDS in resolving a high density of vibrational modes is highlighted by comparing the results obtained here with previous $\mathrm{THz}$ measurements of conventional pellet samples of RDX (Ref. 7) and TNT, ${ }^{1,5,6}$ and a THz measurement of a single RDX crystal. ${ }^{9}$ The pellet samples in these previous works were formed under pressure from a mixture of the explosive powder diluted in a transparent polyethylene matrix. The top panels of Figs. 2(a) and 2(b) show the amplitude absorbance spectra for the RDX film on $\mathrm{Al}$ (at $13 \mathrm{~K}$ ) and the TNT film on Au (at $12 \mathrm{~K}$ ), respectively. In both cases, a broad absorption background has been removed from the spectrum. ${ }^{14}$ The bottom panels show absorbance spectra for RDX (Ref. 7) and TNT pellet samples ${ }^{1,5,6}$ and RDX single crystal. ${ }^{9}$ This comparison emphasizes the much sharper absorption features and much more informative vibrational spectra provided by the waveguide film when cooled to near $10 \mathrm{~K}$. For example, the relatively broad room temperature absorption at $1.06 \mathrm{THz}$ of the RDX Al waveguide film with an FWHM linewidth estimated to be approximately $0.12 \mathrm{THz}$ (Ref. 20) narrows by more than an order of magnitude when the film is cooled to $13 \mathrm{~K}$, producing a blueshifted line at $1.276 \mathrm{THz}$ with a FWHM linewidth of $9 \mathrm{GHz}$ (Table I). In contrast, the corresponding line in the RDX pellet narrows by approximately a factor of 2 upon cooling to $5 \mathrm{~K}$, presumably due to the presence of residual inhomogeneous broadening in the pellet. The significant spectral overlap between broad absorption features in the RDX pellet, even at $5 \mathrm{~K}$, makes it difficult to identify the underlying vibrational modes. In contrast, up to 19 vibrational lines can be identified with waveguide THz-TDS. Similar observations are also made in the case of TNT [Fig. 2(b)], where previous work with pellets ${ }^{1,5,6}$ (at room temperature) has measured up to seven broad lines between 0.5 and $3.5 \mathrm{THz}$, compared to 24 lines (Table I) measured for the film with low temperature waveguide THz-TDS.

From Fig. 2(a), we note that the RDX line frequencies measured by waveguide THz-TDS at $13 \mathrm{~K}$ are blueshifted with respect to those of the RDX pellet ${ }^{7}$ measured at $5 \mathrm{~K}$. We have observed a similar effect for dinitrotoluene isomers when comparing $\mathrm{THz}$ spectra of waveguide films and corresponding pellets, where both samples were characterized near $10 \mathrm{~K}$ in the same cryogenic apparatus. ${ }^{17}$ It is important to determine the origin of this effect, whether it is related to differences in the crystalline properties of the analyte in the 

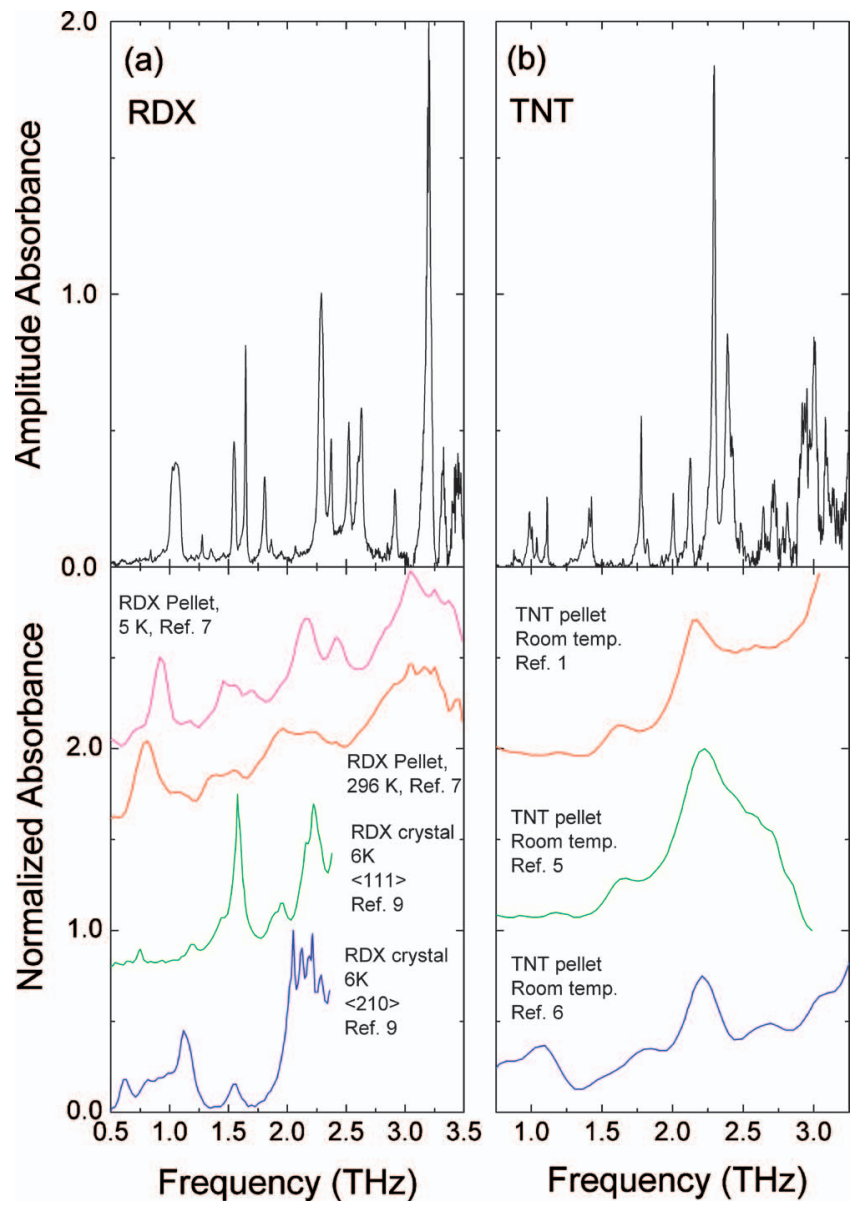

FIG. 2. (Color) A comparison of waveguide THz-TDS measurements of RDX and TNT films with previous THz characterizations of RDX and TNT pellets, and a RDX single crystal. (a) Top panel: amplitude absorbance spectrum of the RDX film on $\mathrm{Al}$ measured in this work at $13 \mathrm{~K}$. Bottom panel: a RDX pellet sample at $5 \mathrm{~K}$ (magenta line) and room temperature (red line) reproduced digitally from Ref. 7, and a RDX single crystal at $6 \mathrm{~K}$ for $\langle 111\rangle$ (green line) and $\langle 210\rangle$ (blue line) orientations, reproduced digitally from Ref. 9. The curves (except the bottom curve) have been offset for clarity. (b) Top panel: amplitude absorbance spectrum of the TNT film on Au measured in this work at $12 \mathrm{~K}$. Bottom panel: three different TNT pellet measurements at room temperature reproduced digitally from Ref. 1 (red line), Ref. 5 (green line), and Ref. 6 (blue line). The curves (except the bottom curve) have been offset for clarity.

film and in the pellet matrix, or possibly due to difficulties in fully cooling pressed pellet samples.

A qualitative comparison to the $\mathrm{THz}$ measurement of the RDX single crystal $^{9}$ at $6 \mathrm{~K}$ [Fig. 2(a), bottom panel] is more difficult because the spectral coverage was limited to about $2.4 \mathrm{THz}$ and the spectral resolution was limited to $1.4 \mathrm{~cm}^{-1}$ $(\sim 47 \mathrm{GHz}){ }^{9}$ Even so, the resolution of individual vibrational absorption lines provided by the RDX waveguide film compares favorably with the $\langle 210\rangle$ and $\langle 111\rangle$ orientations measured for the RDX single crystal. ${ }^{9}$ In addition, we note that the longer temporal scans (up to $133 \mathrm{ps)} \mathrm{with} \mathrm{high} \mathrm{fre-}$ quency resolution necessary for measuring the slowly decaying ringing structure observed in waveguide THz-TDS incorporate additional noise compared to shorter scans with lower frequency resolution.

Optical micrographs taken for RDX and TNT waveguide films ${ }^{20}$ confirm the planar ordering and unique polycrystalline texture formed by each explosive. We have shown that these ordered films are of sufficient crystalline quality to achieve the unprecedented resolution of infrared-active low frequency vibrational modes of explosive solids demonstrated in this work. Further, the relatively small sample mass used in our experiment $(\sim 150 \mu \mathrm{g}$ for RDX) is approximately $1 \%$ of the sample mass typically used in standard pellet measurements of explosives ${ }^{2,5}$ and emphasizes the high sensitivity achieved by waveguide THz-TDS ${ }^{11,13}$ with commercially available standards solutions of these explosives. This is an important safety feature in addition to the ease of sample preparation. The ability of waveguide THzTDS to measure the underlying low frequency vibrational spectrum of explosives together with its high sensitivity shows its potential for applications to THz-based sensing of threat materials. Finally, the interpretation of the waveguide THz-TDS spectra presented here, in terms of assigning the vibrational lines to specific vibrational motion, awaits application of advanced solid state modelling methods.

This work was supported by the Office of Naval Research and the National Science Foundation.

${ }^{1}$ M. C. Kemp, P. F. Taday, B. E. Cole, J. A. Cluff, A. J. Fitzgerald, and W. R. Tribe, Proc. SPIE 5070, 44 (2003).

${ }^{2}$ Y. C. Shen, T. Lo, P. F. Taday, B. E. Cole, W. R. Tribe, and M. C. Kemp, Appl. Phys. Lett. 86, 241116 (2005).

${ }^{3}$ J. F. Federici, B. Schulkin, F. Huang, D. Gary, R. Barat, F. Oliveira, and D. Zimdars, Semicond. Sci. Technol. 20, S266 (2005).

${ }^{4}$ Y. Hu, P. Huang, L. Guo, X. Wang, and C. Zhang, Phys. Lett. A 359, 728 (2006).

${ }^{5}$ J. Chen, Y. Chen, H. Zhao, G. J. Bastiaans, and X.-C. Zhang, Opt. Express 15, 12060 (2007).

${ }^{6}$ M. R. Leahy-Hoppa, M. J. Fitch, X. Zheng, L. M. Hayden, and R. Osiander, Chem. Phys. Lett. 434, 227 (2007).

${ }^{7}$ A. D. Burnett, W. H. Fan, P. C. Upadhya, J. E. Cunningham, H. G. M. Edwards, J. Kendrick, T. Munshi, M. Hargreaves, E. H. Linfield, and A. G. Davies, Proc. SPIE 6549, 654905 (2007).

${ }^{8}$ W. H. Fan, A. Burnett, P. C. Upadhya, J. Cunningham, E. H. Linfield, and A. G. Davies, Appl. Spectrosc. 61, 638 (2007).

${ }^{9}$ J. Barber, D. E. Hooks, D. J. Funk, R. D. Averitt, A. J. Taylor, and D. Babikov, J. Phys. Chem. A 109, 3501 (2005).

${ }^{10}$ J. Zhang and D. Grischkowsky, Opt. Lett. 19, 1617 (2004).

${ }^{11}$ J. S. Melinger, N. Laman, S. S. Harsha, and D. Grischkowsky, Appl. Phys. Lett. 89, 251110 (2006).

${ }^{12}$ R. Mendis and D. Grischkowsky, Opt. Lett. 26, 846 (2001).

${ }^{13}$ G. Gallot, S. P. Jamsion, R. W. McGowan, and D. Grischkowsky, J. Opt. Soc. Am. B 17, 851 (2000).

${ }^{14}$ J. S. Melinger, N. Laman, S. S. Harsha, S. Cheng, and D. Grischkowsky, J. Phys. Chem. A 111, 10977 (2007).

${ }^{15}$ N. Laman, S. S. Harsha, D. Grischkowsky, and J. S. Melinger, Biophys. J. 94, 1010 (2008).

${ }^{16}$ N. Laman, S. S. Harsha, and D. Grischkowsky, Appl. Spectrosc. 62, 319 (2008).

${ }^{17}$ N. Laman, S. S. Harsha, D. Grischkowsky, and J. S. Melinger, Opt. Express 16, 4094 (2008).

${ }^{18}$ D. Plusquellic, K. Siegrist, E. J. Heilweil, and O. Esenturk, ChemPhysChem 8, 2412 (2007).

${ }^{19}$ D. G. Allis, D. A. Prokhorova, and T. M. Korter, J. Phys. Chem. A 110, 1951 (2006).

${ }^{20}$ See EPAPS Document No. E-APPLAB-92-049825 for supplementary material. For more information on EPAPS, see, http://www.aip.org/pubservs/ epaps.html.

${ }^{21}$ R. Ouillon, P. Ranson, and S. Califano, Chem. Phys. 91, 119 (1984).

${ }^{22}$ C. S. Choi and E. Prince, Acta Crystallogr., Sec. B: Struct. Crystallogr. Cryst. Chem. B28, 2857 (1972).

${ }^{23}$ R. M. Vrcelj, J. N. Sherwood, A. R. Kennedy, H. G. Gallagher, and T. Gelbrich, Cryst. Growth Des. 3, 1027 (2003). 